\title{
Porosity of the free boundary for quasilinear parabolic variational problems
}

\author{
Jun Zheng ${ }^{1 *}$, Binhua Feng ${ }^{2}$ and Peihao Zhao ${ }^{3}$
}

"Correspondence:

zhengjun2014@aliyun.com

1 Department of Basic Course, Southwest Jiaotong University, Emeishan, 614202, China Full list of author information is available at the end of the article

\begin{abstract}
In this paper we consider a certain quasilinear parabolic variational problem with identically zero constraint. By using intrinsic scaling, the exact growth of the solution near the free boundary is established. A consequence of this is that the time level of the free boundary is porous (in $N$ dimensions) and therefore its Hausdorff dimension is less than $N$. In particular, the $N$-dimensional Lebesgue measure of the free boundary is zero for each time level.
\end{abstract}

MSC: 35K86; 35K92; 35K65; 35K67

Keywords: parabolic equation; obstacle problem; free boundary; porosity

\section{Introduction and main theorem}

In this paper we consider a variational inequality for the quasilinear parabolic operator

$$
\operatorname{div} a(x, \nabla u)-\partial_{t} u
$$

giving rise to a free boundary. Our purpose is to analyze the free boundary for a large class of obstacle problems associated with degenerate $(2<p<\infty)$ and non-degenerate $(1<p \leq 2)$ parabolic equations. Therefore, let us start with the formulation of the problem in the weak sense. Let $\Omega$ be an open bounded domain of $\mathbb{R}^{N}(N \geq 2), \Omega_{T}=\Omega \times(0, T)$. Denote the parabolic space by $V^{1, p}\left(\Omega_{T}\right)$, see [1],

$$
V^{1, p}\left(\Omega_{T}\right)=L^{\infty}\left(0, T ; L^{1}(\Omega)\right) \cap L^{p}\left(0, T ; W^{1, p}(\Omega)\right) \quad(1<p<\infty) .
$$

The Steklov average $v_{h}$ of a function is defined by

$$
v_{h}(x, t)=\frac{1}{h} \int_{t}^{t+h} v(x, \tau) \mathrm{d} \tau \quad \text { for } t \in(0, T-h]
$$

and $v_{h}=0$ for $t>T-h$. Let the function $a(x, \eta): \Omega \times \mathbb{R}^{N} \rightarrow \mathbb{R}^{N}$ be Lipschitz continuous in $x \in \Omega$ and continuously differentiable in $\eta \in \mathbb{R}^{N} \backslash\{0\}$. Given bounded functions $f, \theta$ and the obstacle 0 , the variational problems are to find a function

$$
u \in \mathcal{K}_{\theta}:=\mathcal{K}_{\theta}(p)=\left\{w: w \in V^{1, p}\left(\Omega_{T}\right), \forall t w=\theta \text { on } \partial_{p} \Omega_{T}, w \geq 0 \text { a.e. in } \Omega_{T}\right\}
$$


where $\partial_{p} \Omega_{T}=(\bar{\Omega} \times\{0\}) \cup(\partial \Omega \times(0, T])$, such that (for $h>0$ and $\left.0<t<t+h<T\right)$

$$
\int_{\Omega} \partial_{t} u_{h}(w-u) \mathrm{d} x+\int_{\Omega}(a(x, \nabla u))_{h} \cdot \nabla(w-u) \mathrm{d} x+\int_{\Omega} f_{h}(w-u) \mathrm{d} x \geq 0
$$

a.e. in $t \in(0, T)$, and for all $w \in \mathcal{K}_{\theta}$.

Under certain conditions on $f$ and $\theta$, we will show that the free boundary of the solution to the variational problems (1) is porous for each $t$-level cut, which implies that the $t$-cuts of the free boundary has Lebesgue measure zero.

As is well known, in the obstacle problems associated with elliptic operators, to obtain the porosity of the free boundary one needs to prove that every solution has a certain growth rate near the free boundary; see [2-4] for instance. When focusing on $p$-parabolic variational problem $(1<p<\infty)$, we remark that due to the lack of the strong minimum principle or the Harnack inequality one cannot inherit each technique from the elliptic obstacle problems, and we need further arguments to establish the growth rate of solutions near the free boundary. In $p$-parabolic variational problems $(p \geq 2)$, Shahgholian overcame this difficulty by using Hölder's estimates for solutions of parabolic equations. As a by-product, the author obtained the porosity of the free boundary for $p \geq 2$; see [5]. A fact that should be noticed is: although neither the technique of Hölder's estimates nor Harnack inequality can be applied to get the growth of solutions in the case of $1<p<2$ in [5], a 'minimum principle (in spatial variables)' for singular parabolic equations given in [6] (Lemma 2.3) may be used in our problem as a substitute tool at this step. Thus in this paper, using the main idea of [5] and techniques of compactness, we are interested in studying the porosity of the free boundary in a large class of variational problems governed by quasilinear parabolic operators. Our result contains not only the case of $p \geq 2$, but the singular case of $1<p<2$ as well, which is naturally an extension of [5].

Throughout this paper, unless specified, we always assume $1<p<\infty$. We make the standard structural conditions on the function $a(x, \eta)$ for some positive constants $\gamma_{0}, \gamma_{1}$, namely,

$\left(\mathrm{a}_{1}\right) a_{i}(x, 0)=0$,

(a $\left.\mathrm{a}_{2}\right) \sum_{i, j=1}^{N} \frac{\partial a_{i}}{\partial \eta_{j}}(x, \eta) \xi_{i} \xi_{j} \geq \gamma_{0}|\eta|^{p-2}|\xi|^{2}$

(a) $\sum_{i, j=1}^{N}\left|\frac{\partial a_{i}}{\partial \eta_{j}}(x, \eta)\right| \leq \gamma_{1}|\eta|^{p-2}$,

(a) $\sum_{i, j=1}^{N}\left|\frac{\partial a_{i}}{\partial x_{j}}(x, \eta)\right| \leq \gamma_{1}|\eta|^{p-1}$,

for a.e. $x \in \Omega$, all $\eta \in \mathbb{R}^{N} \backslash\{0\}$, and all $\xi \in \mathbb{R}^{N}$.

Remark 1.1 Assumptions $\left(a_{1}\right)-\left(a_{4}\right)$ imply that (see $[7,8]$ for instance)

$$
\begin{aligned}
& a(x, \eta) \eta \geq \frac{\gamma_{0}}{p-1}|\eta|^{p}, \\
& |a(x, \eta)| \leq \frac{\gamma_{1}}{p-1}|\eta|^{p-1}, \\
& \left\langle a\left(x, \eta_{1}\right)-a\left(x, \eta_{2}\right), \eta_{1}-\eta_{2}\right\rangle \geq 0,
\end{aligned}
$$

for a.e. $x \in \Omega$ and all $\eta, \eta_{1}, \eta_{2} \in \mathbb{R}^{N}$. Thus the structural conditions for quasilinear operators in [1] are satisfied, which are needed in this paper. 
Suppose that $f$ and $\theta$ are bounded continuous functions on the closure of $\Omega_{T}$. To establish the results obtained in this paper, further conditions on $f$ and $\theta$ are imposed as follows.

(f) $0<\lambda_{0} \leq f \leq \Lambda$ in $\Omega_{T}, f(x, t)$ is monotone non-increasing in $t$;

( $\theta) \theta(x, 0)=0, \theta(x, t)$ is monotone non-decreasing in $t$.

Let us gather some properties (needed here) for the solution $u$ to the variational inequality (1). The following theorem can be proven by classical techniques; we refer the reader to $[2,4,5]$ for sketches of the proofs.

Classical theorem There exists a unique solution $u$ to the variational problem (1) in $\mathcal{K}_{\theta}$ with

$$
\begin{aligned}
& 0 \leq u \leq\|\theta\|_{\infty, \Omega_{T}} \text { in } \Omega_{T}, \\
& \partial_{t} u \geq 0 \quad \text { in }\{u>0\} .
\end{aligned}
$$

Moreover, $u$ satisfies

$$
\operatorname{div} a(x, \nabla u)-\partial_{t} u=g \quad \text { in }\{u>0\} .
$$

weakly in $\Omega_{T}$ with $g \in L^{\infty}\left(\Omega_{T}\right)$ satisfying

$$
f \chi_{\{u>0\}} \leq g \leq f \chi_{\{u>0\}} \text { a.e. in } \Omega_{T} .
$$

We recall the concept of porosity; see $[2,5]$.

Porosity $A$ set $E$ in $\mathbb{R}^{N}$ is called porous with porosity constant $\delta$ if there is an $r_{0}>0$ such that for each $x \in E$ and $0<r<r_{0}$ there is a point $y$ such that $B_{\delta r}(y) \subset B_{r}(x) \backslash E$.

According to [9], a porous set has Hausdorff dimension not exceeding $N-C \delta^{N}$; thus, it is of Lebesgue measure zero.

Now we state the main theorem in this paper.

Theorem 1.1 Let $u$ be the solution to problem (1) in $\mathcal{K}_{\theta}$. Then for every compact set $K \subset \Omega_{T}$

$$
c_{0} r^{\frac{p}{p-1}} \leq \sup _{B_{r}\left(x_{0}\right)} u\left(\cdot, t_{0}\right) \leq C_{0} r^{\frac{p}{p-1}}, \quad \forall\left(x_{0}, t_{0}\right) \in \partial\{u>0\} \cap K .
$$

Furthermore, the intersection $\partial\{u>0\} \cap K \cap\left\{t=t_{0}\right\}$ is porous (in $\mathbb{R}^{N}$ ) with the porosity constant

$$
\delta=\delta\left(\|\theta\|_{\infty, \Omega_{T}}, \lambda_{0}, \Lambda_{0}, \operatorname{dist}\left(K, \partial_{p} \Omega_{T}\right), \gamma_{0}, \gamma_{1}, p\right)
$$




\section{A class of functions on the unit cylinder}

We first let $q=\frac{p}{p-1}$ and $Q_{r}(z, s)=B_{r}(z) \times\left(-r^{q}+s, r^{q}+s\right)$ be the cylinder in $\mathbb{R}^{N+1}$. Write $Q_{1}=$ $Q_{1}(0,0)$, the unit cylinder. Due to the local character of the results obtained in this paper (Theorem 1.1), we may consider the following local formulation. We say that a function $u$ is in $W^{1, p}\left(Q_{1}\right)$ belongs to the class $\mathscr{G}_{a}=\mathscr{G}_{a}\left(p, \gamma_{0}, \gamma_{1}\right)$ if

$$
\begin{aligned}
& \left\|\operatorname{div} a(x, \nabla u)-\partial_{t} u\right\|_{\infty, Q_{1}} \leq 1 ; \\
& 0 \leq u \leq 1, \quad \text { a.e. in } Q_{1} ; \\
& u(0,0)=0 ; \\
& \partial_{t} u \geq 0 \quad \text { a.e. in } Q_{1} .
\end{aligned}
$$

Condition (2a) should be understood in the weak sense, i.e., $\operatorname{div} a(x, \nabla u)-\partial_{t} u=h$ weakly for $h \in L^{\infty}\left(Q_{1}\right)$ with $\|h\|_{\infty, Q_{1}} \leq 1$. Condition (2c) makes sense since (2a) and (2b) provide that $u \in C_{x}^{1, \alpha} \cap C_{t}^{0, \alpha}\left(Q_{\frac{1}{2}}\right)$ and $u \in C^{1, \alpha}\left(Q_{\frac{1}{2}}\right)$ for some $\alpha \in(0,1)$ in the case of $p \geq 2$ and $1<p<2$, respectively (see e.g. Chapter IX of [1]).

In this section, we discuss the behavior of solutions to problem (1) and functions in $\mathscr{G}_{a}$ near the free boundary.

\subsection{Non-degeneracy of the solution near the free boundary}

The following result gives a description of the solution $u$ to problem (1) showing that it cannot grow too slowly near the free boundary. This property and the growth rate of the elements in $\mathscr{G}_{a}$ will pave the way to establish the porosity of the free boundary.

Lemma 2.1 Let $u \in W^{1, p}\left(Q_{1}\right)$ be a non-negative continuous function in $Q_{1}$, satisfying

$$
\operatorname{div} a(x, \nabla u)-\partial_{t} u=f
$$

weakly in $U^{+}=\{u>0\}$. Then for every $(z, s) \in \overline{U^{+}}$and $r>0$ with $Q_{r}(z, s) \subset Q_{1}$

$$
\sup _{(x, t) \in \partial_{p} Q_{r}^{-}(z, s)} u(x, t) \geq c_{0} r^{\frac{p}{p-1}}+u(z, s)
$$

where $Q_{r}^{-}(z, s)=B_{r}(z) \times\left(s-r^{q}, s\right), c_{0}$ is a positive constant depending only on $p, \lambda_{0}, \gamma_{1}$.

Proof First suppose that $(z, s) \in U^{+}$, and for small $\varepsilon>0$ set

$$
u_{\varepsilon}(x, t)=u(x, t)-(1-\varepsilon) u(z, s)
$$

and

$$
v(x, t)=C_{1}|x-z|^{\frac{p}{p-1}}-C_{2}(t-s),
$$

where $C_{1}, C_{2}$ are positive constants, depending only on $p, \lambda_{0}, \gamma_{1}$, such that

$$
\gamma_{1} C_{1}^{p-1}\left(\frac{p}{p-1}\right)^{p}+C_{2} \leq \lambda_{0} .
$$


We claim that for $C_{1}, C_{2}$

$$
\operatorname{div} a(x, \nabla v)-\partial_{t} v \leq \lambda_{0}, \quad \forall(x, t) \in U^{+} \cap Q_{r}^{-}(z, s)
$$

To prove (3), we need to calculate $\nabla v$ and the divergence of $a(x, \nabla v)$. Indeed,

$$
\nabla v(x, t)=\frac{p C_{1}}{p-1}|x-z|^{\frac{2-p}{p-1}}(x-z), \quad\left|D_{i j} v(x, t)\right| \leq \frac{p C_{1}}{(p-1)^{2}}|x-z|^{\frac{2-p}{p-1}}
$$

One may verify that

$$
\begin{aligned}
\operatorname{div} a(x, \nabla v)-\partial_{t} v & =\sum_{i=1}^{N} \frac{\partial a_{i}}{\partial x_{i}}(x, w)+\sum_{i, j=1}^{N} \frac{\partial a_{i}}{\partial \eta_{j}}(x, w) \frac{\partial w_{j}}{\partial x_{i}}(x)+C_{2} \\
& \leq \gamma_{1}|w|^{p-1}+\gamma_{1}|w|^{p-2} \frac{p C_{1}}{(p-1)^{2}}|x-z|^{\frac{2-p}{p-1}}+C_{2} \\
& \leq \gamma_{1}\left(\frac{p C_{1}}{p-1}\right)^{p-1}\left(|x-z|+\frac{1}{p-1}\right)+C_{2} \\
& \leq \gamma_{1}\left(\frac{p C_{1}}{p-1}\right)^{p-1}\left(1+\frac{1}{p-1}\right)+C_{2} \\
& \leq \lambda_{0},
\end{aligned}
$$

where $w(x, t)=\nabla v(x, t)=\frac{p C_{1}}{p-1}|x-z|^{\frac{2-p}{p-1}}(x-z)$.

Notice that $\operatorname{div} a(x, \nabla u)-\partial_{t} u=\operatorname{div} a\left(x, \nabla u_{\varepsilon}\right)-\partial_{t} u_{\varepsilon}$ in $U^{+} \cap Q_{r}^{-}(z, s)$. Recall condition (f), it follows that

$$
\operatorname{div} a(x, \nabla v)-\partial_{t} v \leq \operatorname{div} a\left(x, \nabla u_{\varepsilon}\right)-\partial_{t} u_{\varepsilon} \quad \text { in } \Omega_{+} \cap Q_{r}^{-}(z, s) .
$$

It is easy to see $u_{\varepsilon}(x, t)=-(1-\varepsilon) u(z, s) \leq 0$ on $\partial U^{+}$and $v(x, t) \geq 0$ for any $t \leq s$, thus $u_{\varepsilon} \leq v$ on $\partial U^{+} \cap Q_{r}^{-}(z, s)$. If also $u_{\varepsilon} \leq v$ on $\partial Q_{r}^{-}(z, s) \cap U^{+}$, then we get by the comparison principle

$$
u_{\varepsilon} \leq v \quad \text { in } Q_{r}^{-}(z, s) \cap U^{+}
$$

But $u_{\varepsilon}(z, s)=\varepsilon u(z, s)>0=v(z, s)$, which is a contradiction. Therefore there exists some point $(y, \tau) \in \partial Q_{r}^{-}(z, s)$ such that

$$
u_{\varepsilon}(y, \tau) \geq v(y, \tau)=c_{0} r^{\frac{p}{p-1}}
$$

where $c_{0}=\min \left\{C_{1}, C_{2}\right\}$. Letting $\varepsilon \rightarrow 0$ we obtain the desired result for all $(z, s) \in U^{+}$, and by continuity for all $(z, s) \in \overline{U^{+}}$.

\subsection{Growth rate of the function $u$ in $\mathscr{G}_{a}$}

In this subsection we prove that every function $u$ in $\mathscr{G}_{a}$ cannot grow too fast near the free boundary but has a growth rate of order $q=\frac{p}{p-1}$ (Theorem 2.1). 
First we define the supremum norm of $u$ over the cylinder $Q_{r}^{-}(z, s)$ as [5] by setting

$$
S(r, u, z, s)=\sup _{x \in Q_{r}^{-}(z, s)} u(x, t), \quad \text { and } \quad S(r, u)=\sup _{x \in Q_{r}^{-}(0,0)} u(x, t) .
$$

For each $u \in \mathscr{G}_{a}$, define the set $\mathbb{M}_{a}(u, z, s)$ by setting

$$
\mathbb{M}_{a}(u, z, s)=\left\{j \in \mathbb{N} ; A S\left(2^{-j-1}, u, z, s\right) \geq S\left(2^{-j}, u, z, s\right)\right\}
$$

where $A=2^{q} \max \left\{1, \frac{1}{c_{0}}\right\}$ with $q=\frac{p}{p-1}$, and $c_{0}$ as in Lemma 2.1. For simplicity, we write $\mathbb{M}_{a}(u)=\mathbb{M}_{a}(u, 0,0)$.

It should be noticed that $\mathbb{M}_{a}(u) \neq \emptyset$ for all $u \in \mathscr{G}_{a}$ since $0 \in \mathbb{M}_{a}(u)$. Indeed, it follows from Lemma 2.1 that $S(1, u) \leq 1=\left(\frac{1}{c_{0} 2^{-q}}\right) c_{0} 2^{-q} \leq\left(\frac{1}{c_{0} 2^{-q}}\right) S\left(2^{-1}, u\right)=A S\left(2^{-1}, u\right)$.

Now we state the growth property of the elements in the class $\mathscr{G}_{a}$.

Theorem 2.1 There is a positive constant $M_{0}=M_{0}\left(p, \gamma_{0}, \gamma_{1}\right)$ such that, for every $u \in \mathscr{G}_{a}$,

$$
|u(x, t)| \leq M_{0}(d(x, t))^{q} \quad \forall(x, t) \in Q_{\frac{1}{2}},
$$

where $d(x, t)=\sup \left\{r ; Q_{r}(x, t) \subset U^{+}\right\}$for $(x, t) \in U^{+}$, and $d(x, t)=0$ otherwise.

To prove this theorem we need the following lemma.

Lemma 2.2 There is a positive constant $M_{1}=M_{1}\left(p, \gamma_{0}, \gamma_{1}\right)$ such that

$$
S\left(2^{-j-1}, u\right) \leq M_{1}\left(2^{-j}\right)^{q},
$$

for all $u \in \mathscr{G}_{a}$ and $j \in \mathbb{M}_{a}(u)$.

Proof Arguing by contradiction, we assume that for every $k \in \mathbb{N}$, there exists $u_{k} \in \mathscr{G}_{a}$ and $j_{k} \in \mathbb{M}_{a}\left(u_{k}\right)$ such that

$$
S\left(2^{-j_{k}-1}, u_{k}\right) \geq k\left(2^{-j_{k}}\right)^{q} .
$$

Observe that by the uniform boundedness of $u_{k}$ and (4) it follows that $j_{k} \rightarrow \infty$ as $k \rightarrow \infty$. Consider the function

$$
\widetilde{u}_{k}(x, t)=\frac{u_{k}\left(2^{-j_{k}} x, \alpha_{k} t\right)}{S\left(2^{-j_{k}-1}, u_{k}\right)}
$$

defined in the unit cylinder, where $\alpha_{k}=\left(2^{-j_{k}}\right)^{p}\left(S\left(2^{-j_{k}-1}, u_{k}\right)\right)^{2-p}$. Note that by (4) we have

$$
\begin{aligned}
\alpha_{k} & \leq \frac{1}{k^{p-1}}\left(S\left(2^{-j_{k}-1}, u_{k}\right)\right)^{p-1} \cdot\left(S\left(2^{-j_{k}-1}, u_{k}\right)\right)^{2-p} \\
& =\frac{1}{k^{p-1}} S\left(2^{-j_{k}-1}, u_{k}\right) \\
& \leq \frac{1}{k^{p-1}} \rightarrow 0 \quad \text { as } k \rightarrow \infty
\end{aligned}
$$


By the definition of $\mathbb{M}_{a}\left(u_{k}\right)$ and $\mathscr{G}_{a}$ it follows that

$$
\begin{aligned}
& 0 \leq \tilde{u}_{k} \leq A \quad \text { in } Q_{1}^{-}, \\
& \sup _{\frac{1}{2}} \tilde{u}_{k} \geq 1 \quad\left(\text { by }(2 \mathrm{~d}) \text { and }\left(2^{-1}\right)^{q} \alpha_{k} \geq\left(2^{-j_{k}-1}\right)^{q}\right), \\
& \tilde{u}_{k}(0,0)=0, \\
& \partial_{t} \tilde{u}_{k} \geq 0 \quad \text { in } Q_{1}^{-} .
\end{aligned}
$$

Now, define for $(x, \eta) \in B_{1} \times \mathbb{R}^{N}$

$$
a^{k}(x, \eta)=\left(\frac{2^{-j_{k}}}{S\left(2^{-j_{k}-1}, u_{k}\right)}\right)^{p-1} \cdot a\left(2^{-j_{k}} x, \frac{S\left(2^{-j_{k}-1}, u_{k}\right)}{2^{-j_{k}}} \eta\right) .
$$

We claim that $a^{k}(x, \eta)$ satisfies the same structural conditions as $a(x, \eta)$ for large $k$. Indeed, letting $s_{k}=\frac{2^{-j_{k}}}{S\left(2^{-j_{k}-1}, u_{k}\right)}$, one may verify directly that

$$
\begin{aligned}
\sum_{i, j=1}^{N} \frac{\partial a_{i}^{k}}{\partial \eta_{j}}(x, \eta) \xi_{i} \xi_{j} & =\sum_{i, j=1}^{N} s_{k}^{p-2} \frac{\partial a_{i}}{\partial \eta_{j}}\left(2^{-j_{k}} x, s_{k}^{-1} \eta\right) \xi_{i} \xi_{j} \\
& \geq \gamma_{0} s_{k}^{p-2}\left|s_{k}^{-1} \eta\right|^{p-2}|\xi|^{2} \\
& =\gamma_{0}|\eta|^{p-2}|\xi|^{2} \\
\sum_{i, j=1}^{N}\left|\frac{\partial a_{i}^{k}}{\partial \eta_{j}}(x, \eta)\right| & =\sum_{i, j=1}^{N} s_{k}^{p-2}\left|\frac{\partial a_{i}}{\partial \eta_{j}}\left(2^{-j_{k}} x, s_{k}^{-1} \eta\right)\right| \\
& \leq \gamma_{1} s_{k}^{p-2}\left|s_{k}^{-1} \eta\right|^{p-2} \\
& =\gamma_{1}|\eta|^{p-2}, \\
\sum_{i, j=1}^{N}\left|\frac{\partial a_{i}^{k}}{\partial x_{j}}(x, \eta)\right| & =\sum_{i, j=1}^{N} s_{k}^{p-1} 2^{-j_{k}}\left|\frac{\partial a_{i}}{\partial x_{j}}\left(2^{-j_{k}} x, s_{k}^{-1} \eta\right)\right| \\
& \leq 2^{-j_{k}} \gamma_{1}|\eta|^{p-1} \\
& \leq \gamma_{1}|\eta|^{p-1} \cdot
\end{aligned}
$$

Now by (2a) and (4) we obtain

$$
\begin{aligned}
\left\|\operatorname{div} a^{k}\left(x, \nabla \tilde{u}_{k}(x, t)\right)-\partial_{t} \tilde{u}_{k}(x, t)\right\|_{\infty} & =2^{-j_{k}} s_{k}^{p-1}\left\|\left(A u_{k}-\partial_{t} u_{k}\right)\left(2^{-j_{k}} x, \alpha_{k} t\right)\right\|_{\infty} \\
& \leq 2^{-j_{k}}\left(\frac{2^{-j_{k}}}{S\left(2^{-j_{k}-1}, u_{k}\right)}\right)^{p-1} \\
& \leq \frac{1}{k^{p-1}} \rightarrow 0 \quad \text { as } k \rightarrow \infty,
\end{aligned}
$$

where $(A u)(x, t)$ is defined by $(A u)(x, t)=\operatorname{div} a(x, \nabla u(x, t))$.

Observe that by (5), for any $M>0$ we have

$$
\left|\frac{\partial a_{i}^{k}}{\partial x_{j}}(x, \eta)\right| \rightarrow 0 \quad \text { as } k \rightarrow \infty
$$


uniformly in $(x, \eta) \in B_{1} \times B_{M}$. Therefore the pointwise limit of $a^{k}(x, \eta)$ does not depend on $x$ :

$$
a^{k}(x, \eta) \rightarrow \widetilde{a}(\eta)
$$

with $\widetilde{a}$ satisfying the same structural conditions as $\left(\mathrm{a}_{1}\right)-\left(\mathrm{a}_{4}\right)$. Then invoking compactness arguments (see Lemma 14.1 on p.75 and 14-(iii) on p.115 of [1]), we deduce that, up to a subsequence, $\widetilde{u}_{k}$ converges locally uniformly in $Q_{1}^{-}$to a function $u$. Moreover, the limit function $u$ satisfies

$$
\begin{array}{lll}
\operatorname{div} \widetilde{a}(\nabla u)-\partial_{t} u=0, & u \geq 0, \quad u(0,0)=0, \\
\sup _{Q_{\frac{1}{2}}^{-}} u \geq 1, \quad \partial_{t} u \geq 0 & \text { in } Q_{1}^{-} .
\end{array}
$$

To get a contradiction, we divide our problem into two cases.

-Case $1(1<p \leq 2)$. In this case, we need the following lemma originating from [6], where the authors stated it for $p$-parabolic equations $(1<p<2)$. One should pay attention to the fact that the proof of the following lemma can be repeated as in [6] with slight modifications. Moreover, the result is valid for $p=2$ since the process is 'stable' as $p \nearrow 2$ so that one may recover the regularity results by letting $p \nearrow 2$ (see the proofs of Theorems 1 and 2, or the remarks in 1-(iii) on p.323 of [6]).

Lemma 2.3 (Theorem 2 [6]) Let $\Omega$ be a region of $\mathbb{R}^{N}, \Omega_{\infty}=\Omega \times(0, \infty)$ and $u \in$ $C\left(0, T ; L^{2}(\Omega)\right) \cap L^{2}\left(0, T ; W^{1, p}(\Omega)\right)$ be any non-negative local solution of

$$
\operatorname{div} \tilde{a}(\nabla u)-\partial_{t} u=0 \quad \text { in } \Omega_{\infty}
$$

Suppose $u\left(x_{0}, t_{0}\right)>0$ for some $\left(x_{0}, t_{0}\right) \in \Omega_{\infty}$. Then, for any ball $B_{\rho}\left(x_{0}\right) \subset \Omega$,

$$
u\left(x, t_{0}\right)>0 \quad \forall x \in B_{\rho}\left(x_{0}\right)
$$

Now notice that $\sup _{B_{\frac{1}{2}}} u(x, 0) \geq 1$ by $\partial_{t} u \geq 0$ and (6). One may find $x_{0} \in B_{\frac{1}{2}}(0)$ such that $u\left(x_{0}, 0\right) \geq \frac{1}{2}$. On the other hand, since, for any $\overline{Q^{\prime}} \subset Q_{1}, u \in C^{1, \alpha}\left(Q^{\prime}\right)$ for some $\alpha \in(0,1)$ (see Chapter IX of [1]), and then Lemma 2.3 gives $u(0,0) \geq \frac{1}{2}$, which is a contradiction. Indeed, in Lemma 2.3, one may let $\rho=\frac{1}{2} \in\left(\left|x_{0}\right|, 1-\left|x_{0}\right|\right)$ and $\Omega=B_{r} \subset B_{1}$ with $r=\frac{\frac{1}{2}+\left|x_{0}\right|+1}{2}$. Therefore $B_{\rho}\left(x_{0}\right) \subset \Omega$ and $0 \in B_{\rho}\left(x_{0}\right)$.

-Case $2(2<p<\infty)$. In this case, due to the lack of a strong minimum principle, we need further discussion to get a contradiction. At this point, we show that $u$ is time independent, i.e.,

$$
\partial_{t} u=0 \quad \text { in } Q_{1}^{-} .
$$

Then $u$ is a nonzero, non-negative harmonic function in the unit ball and it vanishes at the origin. Indeed, this is a contradiction to the strong minimum principle; see [10] for instance. To this end, choosing $(x, t),\left(x^{\prime}, t^{\prime}\right) \in Q_{\frac{1}{2}}^{-}$and using the definition of $\mathbb{M}_{a}\left(u_{k}\right)$ and 
Hölder's estimates for solutions with $G_{T}=Q_{2^{-j_{k}}}^{-}$and $K=Q_{2^{-j_{k}-1}}^{-}$(see Theorem 1.1 on p.41 of [1]), we arrive at

$$
\begin{aligned}
\left|\widetilde{u}_{k}(x, t)-\widetilde{u}_{k}\left(x, t^{\prime}\right)\right| & =\frac{\left|u_{k}\left(2^{-j_{k}} x, \alpha_{k} t\right)-u_{k}\left(2^{-j_{k}} x, \alpha_{k} t^{\prime}\right)\right|}{S\left(2^{-j_{k}-1}, u_{k}\right)} \\
& \leq A \frac{\left|u_{k}\left(2^{-j_{k}} x, \alpha_{k} t\right)-u_{k}\left(2^{-j_{k}} x, \alpha_{k} t^{\prime}\right)\right|}{S\left(2^{-j_{k}}, u_{k}\right)} \\
& \leq A \gamma \frac{\left\|u_{k}\right\|_{\infty, G_{T}}}{S\left(2^{-j_{k}, u_{k}}\right)}\left(\frac{\left\|u_{k}\right\|_{\infty, G_{T}}^{\frac{p-2}{p}} \alpha_{k}^{\frac{1}{p}}\left|t-t^{\prime}\right|^{\frac{1}{p}}}{\operatorname{dist}_{p}\left(K, \partial_{p} G_{T} ; p\right)}\right)^{\alpha},
\end{aligned}
$$

where $\operatorname{dist}_{p}\left(K, \partial_{p} G_{T} ; p\right)=\inf _{(x, t) \in K,(y, s) \in \partial_{p} G_{T}}\left(|x-y|+\|u\|_{\infty, G_{T}}^{\frac{2-p}{p}}|t-s|^{\frac{1}{p}}\right), \alpha \in(0,1)$ is the Hölder exponent, the constant $\gamma$ does not depend on $\left\|u_{k}\right\|_{\infty, G_{T}}$.

Now observe that $\operatorname{dist}_{p}\left(K, \partial_{p} G_{T} ; p\right) \geq\left(2^{-j_{k}-1}\right)^{\frac{q}{p}}\|u\|_{\infty, G_{T}}^{\frac{2-p}{p}}$. It follows that

$$
\begin{aligned}
\left|\widetilde{u}_{k}(x, t)-\widetilde{u}_{k}\left(x, t^{\prime}\right)\right| & \leq A \gamma\left|t-t^{\prime}\right|^{\frac{\alpha}{p}}\left(\alpha_{k} \cdot 2^{\left(j_{k}+1\right) q}\right)^{\frac{\alpha}{p}} \\
& =A \gamma\left|t-t^{\prime}\right|^{\frac{\alpha}{p}}\left[\left(2^{-j_{k}}\right)^{p} \cdot\left(S\left(2^{-j_{k}-1}, u_{k}\right)\right)^{2-p} \cdot 2^{\left(j_{k}+1\right) q}\right]^{\frac{\alpha}{p}} \\
& \leq A \gamma\left|t-t^{\prime}\right|^{\frac{\alpha}{p}}\left[\left(2^{-j_{k}}\right)^{p-1} \cdot k^{2-p} \cdot\left(2^{-j_{k}}\right)^{q(2-p)} \cdot\left(2^{j_{k}}\right)^{q} \cdot 2^{q}\right]^{\frac{\alpha}{p}} \\
& =2^{\frac{\alpha}{p-1}} A \gamma\left|t-t^{\prime}\right|^{\frac{\alpha}{p}} k^{2-p} \rightarrow 0 \quad \text { as } k \rightarrow \infty .
\end{aligned}
$$

Hence $u$ is $t$-independent, and the proof is completed.

Proof of Theorem 2.1 The proof of this theorem is standard (see [5]). For convenience, we recover the process. Let us take $j$ for which

$$
S\left(2^{-j}, u\right)>2^{q} M_{1} 2^{-q j} .
$$

It follows that

$$
S\left(2^{-j+1}, u\right) \leq 2^{q} M_{1} 2^{-q(j-1)}<2^{q} S\left(2^{-j}, u\right) \leq A S\left(2^{-j}, u\right),
$$

i.e. $j-1 \in \mathbb{M}_{a}(u)$, so Lemma 2.2 holds for $j-1$. Now we arrive at the following obvious contradiction to (7):

$$
S\left(2^{-j}, u\right) \leq S\left(2^{-j+1}, u\right) \leq M_{1} 2^{-q(j-1)}=2^{q} M_{1} 2^{-q j} .
$$

Therefore

$$
S\left(2^{-j}, u\right) \leq 2^{q} M_{1} 2^{-q j}, \quad \forall j,
$$

which implies

$$
\sup _{Q_{r}^{-}(0,0)} u \leq 2^{q} M_{1} 2^{-q j}, \quad \forall r \leq 1 .
$$


To obtain a similar estimate for $u$ over the whole cylinder (and not only over the lower half part) we use an upper barrier. Define $w(x, t)=C_{3}|x|^{q}+C_{4} t$ where $C_{4}=1+\gamma_{1}\left(q C_{3}\right)^{p-1}(1+$ $\left.\frac{1}{p-1}\right)$ and $C_{3}>0$. Let now $Q_{1}^{+}=B_{1}(0) \times(0,1)$. Then proceeding as Lemma 2.1, we deduce

$$
\begin{aligned}
\operatorname{div} a(x, \nabla w)-\partial_{t} w & \leq \gamma_{1}\left(q C_{3}\right)^{p-1}\left(1+\frac{1}{p-1}\right)-C_{4} \\
& =-1 \leq \operatorname{div} a(x, \nabla u)-\partial_{t} u \quad \text { in } Q_{1}^{+} .
\end{aligned}
$$

Since by choosing $C_{3}$ large, we will have $w \geq u$ on $\partial_{p} Q_{1}^{+}$, where for the estimate on $\{t=0\}$ we have sued the previous discussion, i.e., $S(r, u) \leq C r^{q}$. Hence by the comparison principle we have $w \geq u$ in $Q_{1}^{+}$. Therefore

$$
\sup _{Q_{r}(0,0)} u \leq M_{2} r^{q}
$$

The proof is completed.

\section{Proof of the main theorem}

Having the estimates from below and above for the function $u$, one can prove our main result as in [5]. For completeness we carry out the minor changes in the proof of [5].

Proof of the main theorem Without loss of generality, we assume that the compact set $K$ in the main theorem is the closed unit cylinder $\bar{Q}_{1}$, and, moreover, that $\bar{Q}_{2} \subset \Omega_{T}$.

For $(x, t) \in U^{+} \cap \bar{Q}_{1}$, let $d(x, t)$ be defined as in Theorem 2.1 and take $\left(x^{0}, t^{0}\right) \in \partial U^{+} \cap$ $\bar{Q}_{1}$ which realizes this distance. Next define $\widetilde{u}(y, s)=u\left(x^{0}+y, t^{0}+s\right)$ in $Q_{1}$. Let $M=$ $\max \left\{\|\theta\|_{\infty, \Omega_{T}}, \Lambda_{0}\right\}, \widetilde{a}(y, \eta)=\frac{a\left(x^{0}+y, M \eta\right)}{M}$ and $\tilde{A} v(y, s)=\operatorname{div} \widetilde{a}(y, \nabla v(y, s))$. We claim that $\frac{\widetilde{u}}{M} \in$ $\mathscr{G}_{\tilde{a}}$. Indeed, one may verify directly that $\tilde{a}$ satisfies all structural conditions (not necessarily with the same constants as $a$ ). Furthermore, we have

$$
\begin{aligned}
\widetilde{A}\left(\frac{\tilde{u}}{M}\right)-\partial_{s}\left(\frac{\tilde{u}}{M}\right) & =\frac{1}{M}\left[\operatorname{div} a\left(x^{0}+y, \nabla u\left(x^{0}+y, t^{0}+s\right)\right)-\partial_{s} u\left(x^{0}+y, t^{0}+s\right)\right] \\
& =\frac{1}{M}\left[(A u)-\partial_{s} u\right]\left(x^{0}+y, t^{0}+s\right) \\
& \leq \frac{\Lambda_{0}}{M} \\
& \leq 1
\end{aligned}
$$

and

$$
0 \leq \frac{\widetilde{u}}{M} \leq \frac{\|\theta\|_{\infty, \Omega_{T}}}{M} \leq 1 \quad \text { and } \quad \frac{\widetilde{u}(0,0)}{M}=0 .
$$

Therefore we infer by Theorem 2.1 that

$$
u(x, t)=\widetilde{u}\left(x-x^{0}, t-t^{0}\right) \leq M M_{0}(d(x, t))^{\frac{p}{p-1}} .
$$

Let $(z, \tau) \in \partial U^{+} \cap \bar{Q}_{1}$. Then, for $0<r<1$, by Lemma 2.1, there exists $x^{1} \in \partial B_{r}(z)$ such that

$$
u\left(x^{1}\right) \geq c_{0} r^{\frac{p}{p-1}} .
$$


It follows from (8) that

$$
c_{0} r^{\frac{p}{p-1}} \leq u\left(x^{1}, \tau\right) \leq M M_{0}(d(x, \tau))^{\frac{p}{p-1}} .
$$

Let $\delta=\left(\frac{c_{0}}{M M_{0}}\right)^{\frac{p-1}{p}}$. Then $d(x, \tau) \geq \delta r$, and $0<\delta \leq 1$. Therefore

$$
B_{\delta r}\left(x^{1}\right) \cap B_{r}(z) \subset U^{+} .
$$

Now choose $y \in\left[z, x^{1}\right]$ such that $\left|y-x^{1}\right|=\frac{\delta r}{2}$. Then we have

$$
B_{\frac{\delta r}{2}}(y) \subset B_{\delta r}\left(x^{1}\right) \cap B_{r}(z) \subset B_{r}(z) \backslash \partial U^{+} .
$$

Indeed, for any $y_{0} \in B_{\frac{\delta r}{2}}(y)$, we have

$$
\left|y_{0}-x^{1}\right| \leq\left|y_{0}-y\right|+\left|y-x^{1}\right|<\frac{\delta r}{2}+\frac{\delta r}{2}=\delta r
$$

Moreover, since $|y-z|=\left|z-x^{1}\right|-\left|y-x^{1}\right|$, we have

$$
\left|y_{0}-z\right| \leq\left|y_{0}-y\right|+\left(\left|z-x^{1}\right|-\left|y-x^{1}\right|\right) \leq \frac{\delta r}{2}+\left(r-\frac{\delta r}{2}\right)=r
$$

This shows that $\partial U^{+} \cap\{t=\tau\} \cap \bar{B}_{1}$ is porous with the porosity constant $\frac{\delta}{2}$.

\section{Competing interests}

The authors declare that they have no competing interests.

\section{Authors' contributions}

The authors contributed equally to this work. The three authors have contributed to the manuscript; they wrote, read, and approved the manuscript.

\section{Author details}

'Department of Basic Course, Southwest Jiaotong University, Emeishan, 614202, China. ${ }^{2}$ College of Mathematics and Statistics, Northwest Normal University, Lanzhou, 730070, China. ${ }^{3}$ School of Mathematics and Statistics, Lanzhou University, Lanzhou, 730000, China.

\section{Acknowledgements}

The authors would like to thank the referee for his/her careful reading and valuable suggestions, which made this article more readable. This work is supported by the Fundamental Research Funds for the Central Universities: 10801B10096018 and $10801 \times 10096022$.

Received: 22 April 2015 Accepted: 24 October 2015 Published online: 02 November 2015

\section{References}

1. DiBenedetto, E: Degenerate Parabolic Equations. Universitext. Springer, New York (1993)

2. Karp, L, Kilpeläinen, T, Petrosyan, A, Shahgholian, H: On the porosity of free boundaries in degenerate variational inequalities. J. Differ. Equ. 164, 110-117 (2000)

3. Zheng, J, Zhang, Z: Porosity of the free boundary in the obstacle problems. J. Shaanxi Normal Univ. Nat. Sci. Ed. 40(2), $11-13,18(2012)$

4. Zheng, J, Zhang, Z, Zhao, P: Porosity of free boundaries in the obstacle problem for quasilinear elliptic equations. Proc. Indian Acad. Sci. Math. Sci. 123(3), 373-382 (2013)

5. Shahgholian, $\mathrm{H}$ : Analysis of the free boundary for the $p$-parabolic variational problem ( $p \geq 2)$. Rev. Mat. Iberoam. 19 797-812 (2003)

6. Chen, Y, DiBenedetto, E: On the local behavior of solutions of singular parabolic equations. Arch. Ration. Mech. Anal. 103(4), 319-345 (1988)

7. DiBenedetto, $\mathrm{E}: C^{1, \alpha}$ Local regularity of weak solutions of degenerate elliptic equations. Nonlinear Anal. 7, 827-850 (1983)

8. Tolksdorf, P: Regularity for a more general class of quasilinear elliptic equations. J. Differ. Equ. 51, 126-150 (1984)

9. Martio, O, Vuorinen, M: Whitney cubes, p-capacity, and Minkowski content. Expo. Math. 5(1), 17-40 (1987)

10. Pucci, P, Serrin, J: The strong maximum principle revisited. J. Differ. Equ. 196, 1-66 (2004) 